\title{
Aesthetic Treatment of Four Maxillary Anterior Teeth With Endodontic Failure: A Case Report
}

\author{
Fransisca Debby Rosalia D ${ }^{1}$, Diatri Nari Ratih ${ }^{2}$, Margareta Rinastiti ${ }^{2}$

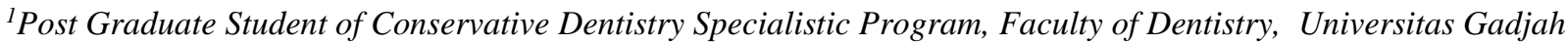 \\ Mada. \\ ${ }^{2}$ Department of Conservative Dentistry, Faculty of Dentistry, Universitas Gadjah Mada \\ *Corresponding author; E-mail: fransiscadebbyrosalia@gmail.com
}

\begin{abstract}
Aesthetic aspect has become increasingly important in the modern restorative dentistry. Restoration, which considering aesthetics and function, is a paramount importance to the patient, especially in anterior region. Aesthetic analysis consists of facial and space analysis of teeth. Case report. A 23-year-old female patient presented to the Clinic of Conservative Dentistry Dental Hospital of Gadjah Mada University complaining a broken restoration of 12 tooth along with acute pain. Two years earlier, the tooth had been treated endodontically and direct restoration performed. Four days ago, the restoration was broken. A clinical examination revealed that teeth 12,11, 21, dan 22 had been previously restored using composite, though it had non-adequate contour and discoloration. Teeth 12 and 11 were tenderness in percussion and enlargement gingiva occurred. A periapical radiograph showed incomplete root canal obturation with apical lesion in teeth 12 and 11. After examination and aesthetic analysis completed, teeth 12 and 11 were carried out root canal retreatment followed by crown lengthening. One week following crown lengthening, teeth 12 and 11 were restored using fiber post and indirect composite crown. Teeth 21 and 22 were restored with direct veneer using composite to achieve ideal width of each tooth. Patient was recalled after two weeks, no complain, asymptomatic teeth and no underfilling were note. Radiographic examination revealed that the diameter of apical lesion became smaller. Conclusion. Aesthetic restoration is important to be conducted following endodontic treatment. Aesthetic restoration is associated with the successful of endodontic treatment in achieving normal function of the teeth.
\end{abstract}

Keywords: retreatment, crown lengthening, indirect composite crown, direct veneer

\section{INTRODUCTION}

Post-endodontic restoration must consider the protection and conservation of the remaining hard tissue of the teeth, the aesthetic conditions and occlusal pressure that affects the teeth. To obtain optimal aesthetic appearance, the dentist must be able to create the appearance of natural teeth with the ideal shape and size, color, and inclination [1]. This is created through an understanding of aesthetic principles. In material selection, the dentist must consider the subjective and objective aspects and expectations of the patient $[1,2]$

The success of root canal treatment depends on the accuracy of diagnosis, treatment planning, the stage of biomechanical preparation, obturation hermetically the root canal system [3].
Root canal treatment is inseparable from the possibility of failure. One of the factors causing the failure of the root canal treatments is the error in treatment procedures, such as cleaning and obturation of root canal. Failure of root canal treatment resulting in the periapical lesions, which in turns, requiring retreatment [4].

The aims of root canal retreatment is to eliminate bacteria and prevent further contamination with hermetic obturation and good restoration [4]. The purpose of this case report was to present the success of root canal retreatment in teeth 12 and 11 with periapical lesions and restoration using direct veneer in teeth 21 and 22 by considering aesthetic analysis. 


\section{CASE REPORT}

\subsection{Case Presentation}

A 23-year-old female patient presented to the Clinic of Conservative Dentistry Dental Hospital of Gadjah Mada University complaining a broken restoration of tooth 12 along with acute pain (Figure 1). Two years earlier, the tooth had been treated endodontically and direct restoration performed. Four days ago, the restoration was broken. A clinical examination revealed that teeth $12,11,21$, and 22 had been previously restored using composite, though it had non-adequate contour and discoloration. Teeth 12 and 11 were tenderness in percussion and enlargement gingiva occurred. A periapical radiograph showed incomplete root canal obturation with apical lesion in teeth \#12 \#11 (Figure 2).

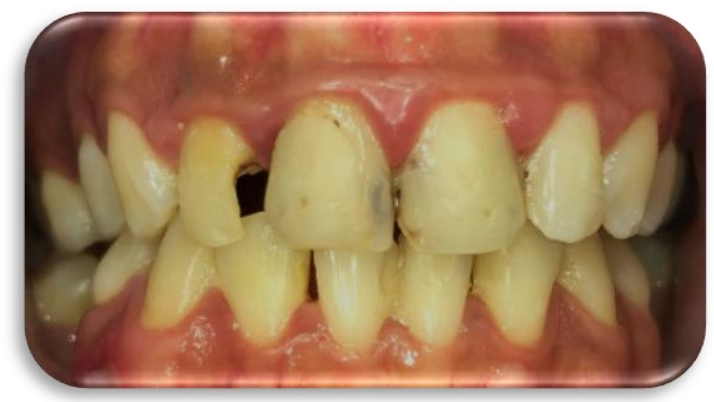

Figure 1. Broken Restoration of 12 tooth

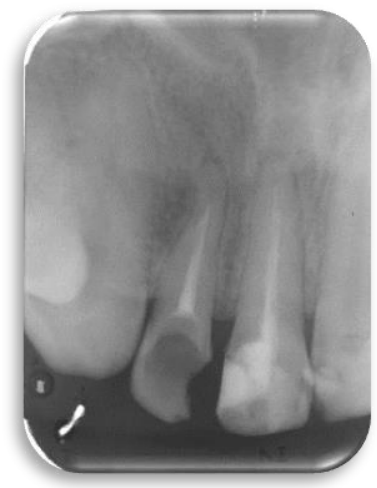

Figure 2. Radiographic examination on teeth 12 and 11

\subsection{Investigations}

Esthetical analysis comprised facial analysis, dentofacial analysis, smile analysis, gingival analysis, and space analysis (Figure 3 and 4). Space analysis showed inter-canine distance of 43,5 mm $(22,6 \mathrm{~mm}$ from right canine to median line and $20,6 \mathrm{~mm}$ from left canine to median line). Based on proportion measurement, the ideal height of teeth 11,21 was 7,5 $\mathrm{mm}$ and ideal tooth width was $6,4 \mathrm{~mm}$ (Figure 5 and $6)$. Based on the golden proportion rule, the ideal width of teeth 12, 22 was $4 \mathrm{~mm}$, therefore teeth mockup was made based on space analysis.

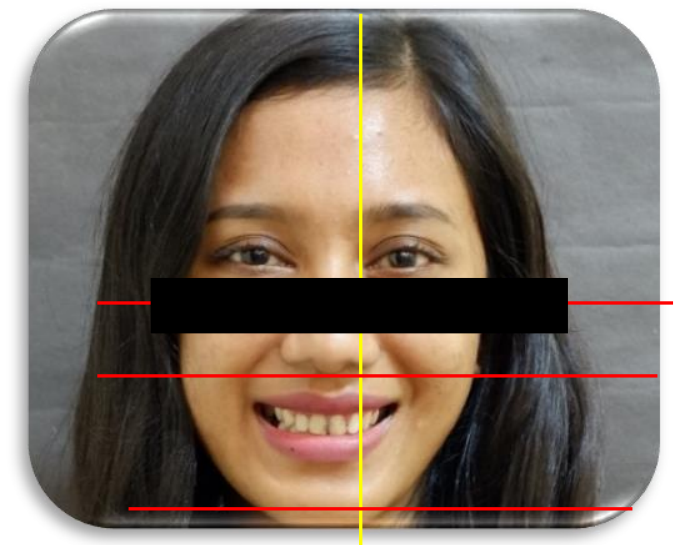

Figure 3. Dentofacial analysis from frontal

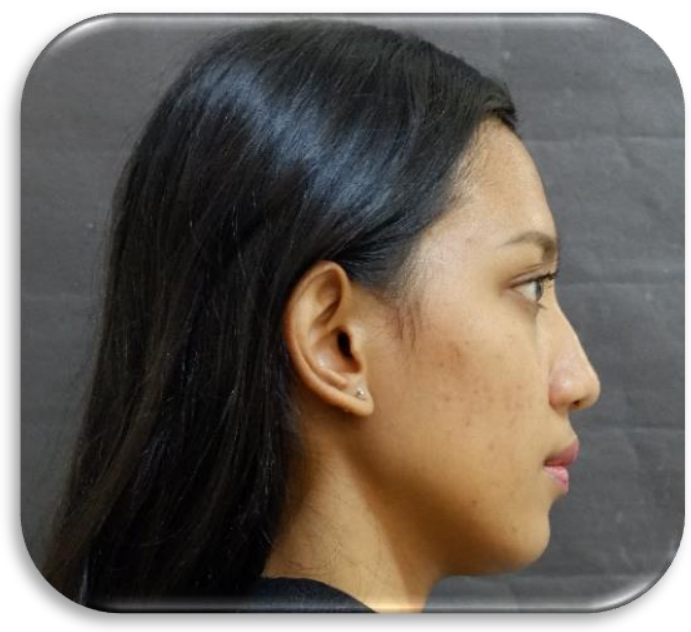

Figure 4. Dentofacial analysis from the side

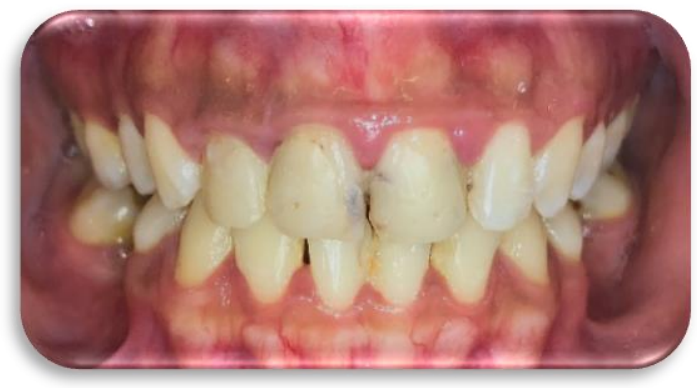

Figure 6. Space Analysis of anterior teeth

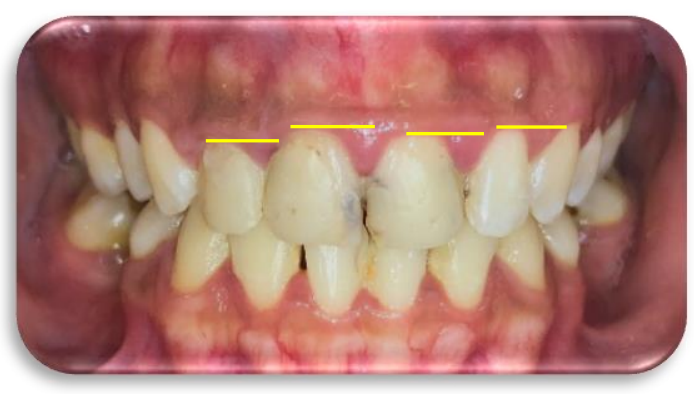

Figure 5. Gingival Zenith position 


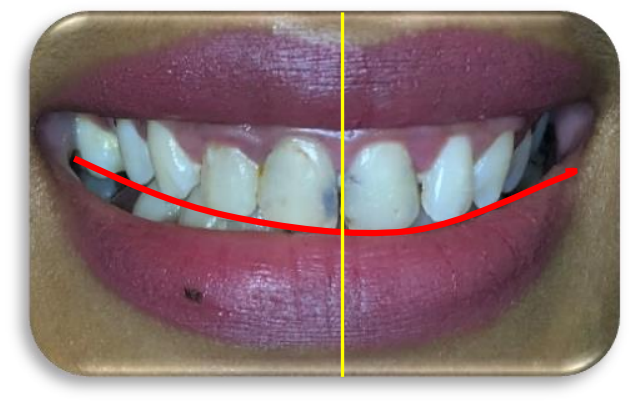

Figure 7. Smile analysis

\subsection{Treatment}

Root canal retreatment was carried out on tooth 12 and tooth 11 under rubber dam isolation. Gutta-percha was removed using $\mathrm{H}$-file. Tooth 12 was restored with composite resin to create artificial wall. Working length was determined using electronic apex locator, and was confirmed with periapical radiograph (Figure 8 and 9). Root canal preparation was performed using crown down technique with rotary file (Protaper Next X1-X5, Dentsply Maillefer, Switzerland). Root canal irrigation was done using 3\% sodium hypochlorite, $17 \%$ EDTA, saline, and final rinse with $2 \%$ Chlorhexidine. Root canals were dried using paper points, then calcium hydroxide was applied as intracanal dressing and cavity was sealed with temporary restoration. Patient recall was scheduled after one week.

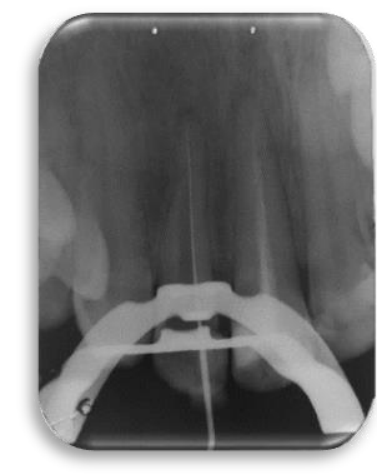

Figure 8. Working length determination by radiograph of tooth 12

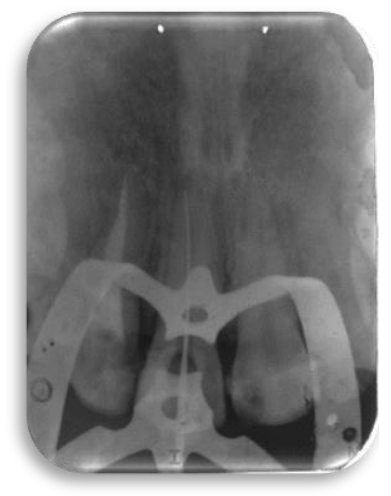

Figure 9. Working length determination by radiograph of tooth 11
Teeth 12 and 11 were asymptomatic on second visit. Master Apical Cone (MAC) of gutta-perhca was inserted in root canal according to working length and confirmed with periapical radiograph. Root canals were obturated using cold lateral condensation technique with epoxy resin sealer followed by temporary filling to seal the cavity, and periapical radiograph was taken (Figure 10).

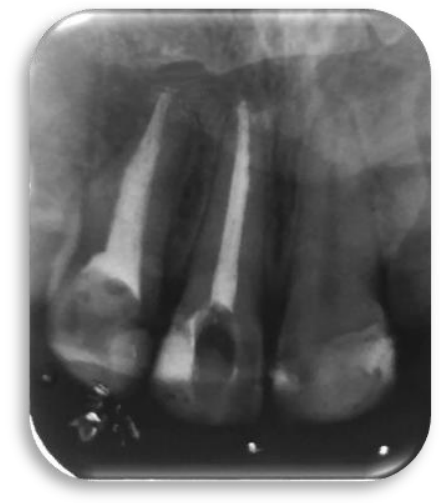

Figure 10. Periapical radiograph immediately after obturation

Gingivectomy was performed on third visit to correct the gingival enlargement on interdental tooth 12 and 11 followed by gingival recontouring on four maxillary anterior teeth for esthetical consideration. Fiber posts were inserted into the canals of teeth 12 and 11 using resin cement (Built It FR, Pentron Clinical,California,USA). Then crown preparation was performed on teeth 12 and 11 for composite resin crown restoration and impression was taken.

Composite resin crowns were inserted on fourth visit followed by direct veneer restoration on teeth 21 and 22 using composite resin (Filtek Z350, 3M ESPE, St.Paul, MN,USA) (Figure11 and 12). Occlusion and marginal adaptation were checked clinically and postoperative radiograph was taken. After one-week patient came for recall, there were no complaints and teeth were functioning normally. Periapical radiograph showed that radiolucency was no longer present.
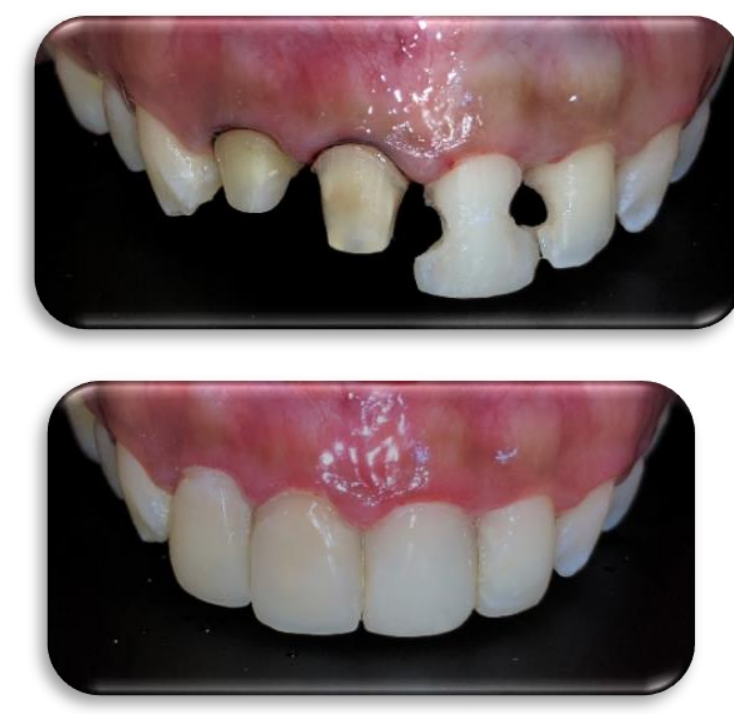

Figure 11. Before cementation crown

Figure 12. After cementation of crown and direct veneer 


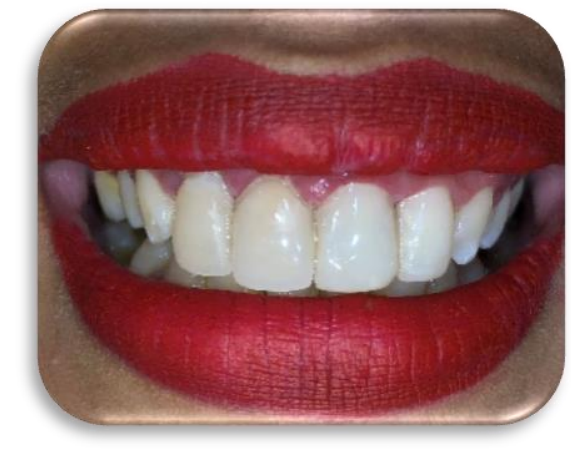

Figure 13. Clinical view after one-week control

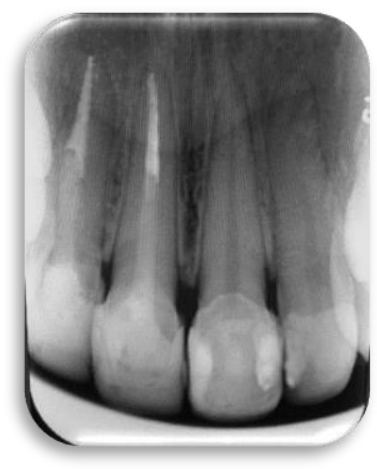

Figure 14. Radiograph view after control

\section{DISCUSSION}

Root canal treated teeth often occurs recurrent pain and periapical pathosis. The cause of pain and pathosis might be due to inflammatory of peri radicular lesion. This complaint occurs because non healing lesion following root canal treatment had been completed [5]. This condition is caused by bacterial infection of the root canal system [6]. Bacteria might resist during the chemo-mechanical cleaning of root canals or might penetrate from leaking restoration. In this case non-surgical retreatment approach was chosen. The goal of root canal retreatment is to remove materials from the root canal, and to repair defects that are pathologic or iatrogenic in origin [7].

It is important to place good restoration on root canal treated teeth. Permanent restoration should be placed as soon as possible after root canal treatment had been completed. Before restoration, it is recommended to seal the canal orifice. The restoration to be chosen depends on the remaining tooth structure and the loading direction. The options for anterior teeth restoration are direct composite filling or indirect crowns with or without a post. Posts can be beneficial to retain core materials to enhance retention and resistance form. In anterior teeth, posts can be useful if a crown will be placed, as the post will allow lateral load to be distributed from the cervical area [8]. If the remaining tooth structure of anterior teeth is minimal to moderate, then a direct composite restoration will be the restoration of choice. It is beneficial to place composite resin below the level of the cemento-enamel junction to provide good coronal seal and reduce susceptibility to fracture. Composite resin restoration offers several advantages, such as good sealing ability, good physical properties and a lot variety of tooth shades, and good aesthetic appearance [9].

Teeth 11 and 12 were restored with composite crowns because this material has a number of advantages. Namely less invasive material, hence more natural tooth structure intact, greater ferrule height and residual wall thickness. Composite do not cause abrasion to antagonizing teeth. Composite materials can also be repaired easily with the similar material. Furthermore, adhesive resin cements are chemically compatible with indirect composite [10]. Another advantage of indirect composite restorations is that the composite is placed in bulk and is polymerized before placement, thus avoiding shrinkage

The concept of dental esthetic harmonizes the facial esthetics with dental facial composition including lips and smile. The dental composition relates more specifically to the size, shape, and positions of the teeth and their relationship to the alveolar bone and gingival tissues. Therefore, smile design includes the evaluation and analysis of hard and soft tissues of the face and smile [11]. Detail aesthetic analysis of smiles can be a standard for improving dental aesthetics by determining smile lines and the amount of teeth and gingiva exposed when smiling. Aesthetic analysis of the gingival aspects is related to the condition of soft tissue around the teeth including gingival texture, gingival shape, and the relationship of teeth to the gingiva. The poor appearance of the gingiva can adversely affect all dental aesthetics [12]. In this case, since the gingiva have an un-aesthetic contour, therefore crown lengthening and gingiva recontouring were carried out.

Teeth 21 and 22 were restored with direct composite resin veneer. The main advantages of this restoration are related to their adhesive properties, the minimal amount of preparation, reinforcement of remaining teeth and the esthetic appearance [13]. Polishing of direct composite veneers is relatively easy and any cracks or fractures of the restoration can be repaired intraorally. In addition, marginal adaptation is better than that of indirect veneer restorations [14]. Besides veneer, it is also recommended to use composite resin with smaller filler size (micro-filled or nano-filled composites) in order to produce a smoother surface and better esthetic appearance [13]. 


\section{CONCLUSION}

Aesthetic restoration is important to be conducted following endodontic treatment. Aesthetic restoration is associated with the successful of endodontic treatment in achieving normal function of the teeth.

\section{REFERENCES}

[1] Summit JB, Robbins JM, Hilton TJSchwartz RS. 2006. Fundamentals of Operative Dentistry: A Contemporary Approach 3 rd ed. Chicago:Quintessence.

[2] Roulet JF. 2003. Indirect Aesrhetics Restoration. J. Adv Aesth Res Dent, 5: 15-9

[3] Estrela, Carlos., Holland,Roberto., Estrela CRDA., Alencar AHG., Sousa-Neto MD., Pecora JD., 2014. Characterization of Successful Root Canal Treatment. Brazilian Dental Journal, 25(1):3-11.

[4] Pinheiro ET, Gomes BPFA, Ferraz CCR, Sousa ELR, Teixeira FB, Souza-Filho FJ. 2003.Mikrorganisms from Canal of RootFilled Teeth with Periapical Lessions. Iternational Endodontic Journal,36:1-11.

[5] Haapasalo M,Shen Y, Ricucci D,2008, Reasons for Persistentand Emerging Post Treatment Endodontic Disease, Endodontics Topics 18,31-50

[6] Priyanka.S.R , Veronica, 2013, Flare-Ups in

Endodontics - A Review, IOSR-JDMS Volume 9, Issue 4 (Sep.- Oct. 2013), PP 26-31

[7] Ruddle CJ, 2004, NONSURGICAL

ENDODONTIC RETREATMENT, CDA JOURNAL.

[8] S. Eliyas, J. Jalili and N. Martin, 2015, Restoration of the root canal treated tooth, British Dental

[1] Journal Vol.218 NO. 2 Jan 232015.

[9] F. Mannocci and J. Cowie1, 2014, Restoration of endodontically treated teeth, British Dental Journal Vol. 216 NO. 6

[10] L.A. Jongsma*, C.J. Kleverlaan, A.J. Feilzer,

2012, Clinical success and survival of indirect resin composite crowns: Results of a 3-year prospective study, Dental Materials 28: 952-960

[11] Davis, Nicholas C., 2007, Smile Design, Dent Clin N Am, 51:299-318.

[12] Ahmad I. 2005.Anterior Dental Aesthetics: Gingival Persepective. Brit Dent J.199(4):195-202

[13] Coelho-de-Souza FH, Goncalves DS, Sales MP, Erhardt MCG, Correa MB, Opdam NJM, Demarco FF, 2015, Direct Anterior Composite Veneers in Vital and Non-Vital Teeth: a Retrospective Clinical Evaluation, Journal of Dentistry.

[14] Sowmya, Kyatham., Dwijendra KS., Pranitha V., Roy, KK., 2017. Esthetic Rehabilitation with Direct Composite Veneering: A report of 2 Cases. International Journal of Dentistry. 